\title{
An unusual case of cutaneous metastases from gallbladder cancer
}

\author{
Ke-Kang Sun ${ }^{1}$, Xiao-Jun Shen ${ }^{1}$, Hua Zhao ${ }^{2}$ \\ ${ }^{1}$ Department of General Surgery, Kunshan First People's Hospital Affiliated to Jiangsu University, Kunshan 215300, China; ${ }^{2}$ Department of General \\ Surgery, First Hospital Affiliated to Soochow University, Suzhou 215006, China \\ Correspondence to: Hua Zhao, PhD. Department of General Surgery, First Hospital Affiliated to Soochow University, Suzhou, Jiangsu 215006, China. \\ Email: hzhaomd@163.com.
}

\begin{abstract}
Cutaneous metastasis of gallbladder cancer is an exceedingly infrequent phenomenon. Herein, we report a case of cutaneous metastases of gallbladder carcinoma as a first clinical manifestation in a 73-yearold woman. Physical examination revealed a violaceous nodule with central ulceration on left upper abdominal wall, ranging in diameter about $3 \mathrm{~cm}$. Biopsy specimen demonstrated middle differentiated carcinoma. Immunohistochemical analysis showed diffuse staining with cytokeratin (CK)-7, CK-19 and carcino-embryonic antigen (CEA). Further computed tomography (CT) and positron emission tomography (PET)/CT scanning confirmed a large occupying lesion in the gallbladder, enlarged lymph nodes in front of the portal vein and behind the common bile duct. The diagnosis of metastatic adenocarcinoma was made. The patient received four-cycle chemotherapy. However, CT scan showed tumor progression with multiple metastatic nodule in lung and subcutaneous nodules. The patient eventually succumbed to the disease less than half a year. As for gallbladder cancer, the skin is an uncommon metastatic site with only a few reported cases. Clinicians should be aware that metastatic cutaneous lesions could be the initial presenting sign for gallbladder cancer.
\end{abstract}

Keywords: Gallbladder cancer; cutaneous metastases

Submitted Mar 24, 2017. Accepted for publication Jul 24, 2017.

doi: $10.21037 /$ tcr.2017.08.11

View this article at: http://dx.doi.org/10.21037/tcr.2017.08.11

\section{Introduction}

Gallbladder cancer is a rarely seen type of malignancy, but it is the most common type of biliary cancer. In general, metastases of gallbladder carcinoma tend to occur in the regional lymph nodes and liver, whereas extra-abdominal and blood-borne metastases are relatively infrequent (1). Cutaneous metastases from primary gallbladder cancer are extremely rare. According to the MEDLINE database, only six cases were documented between 1961 and 2014, apart from fine needle aspiration cytology site or laparoscopic incision (2-7). An unusual case of cutaneous metastases is the initial presentation. Subsequently, multiple subcutaneous nodule and lung metastases of gallbladder cancer without active disease appear in the abdomen or at the original site.

\section{Case presentation}

A 73-year-old woman developed subcutaneous abscess on the upper abdominal wall over a period of 2 weeks. Skin lesions were initially diagnosed as pyogenic granulomas, and hence incised, drained, and treated with antibiotics for suppurative infection without pathological examination. The incised areas became uplift ulcers and failed to heal. Physical examination revealed a violaceous nodule (approximately $3 \mathrm{~cm}$ in diameter) with central ulceration on the left upper abdominal wall. Abdominal wall biopsy confirmed mildly differentiated metastatic adenocarcinoma of the skin. Immunohistochemical analysis showed diffuse staining with cytokeratin (CK)-7, CK-19, and carcinoembryonic antigen (CEA) (Figure 1). Further computed tomography (CT) and ultrasound scanning confirmed a massive lesion occupying the gallbladder and enlarged lymph nodes in front of the portal vein (Figure 2A). Positron emission tomography (PET)/CT demonstrated a fluorodeoxyglucose-reactive gallbladder with maximum standardized uptake value $\left(\mathrm{SUV}_{\text {max }}\right)$ of 11.4 and left upper abdominal wall metastases $\left(\mathrm{SUV}_{\text {max }}: 6.2\right)$. The patient was diagnosed with skin 

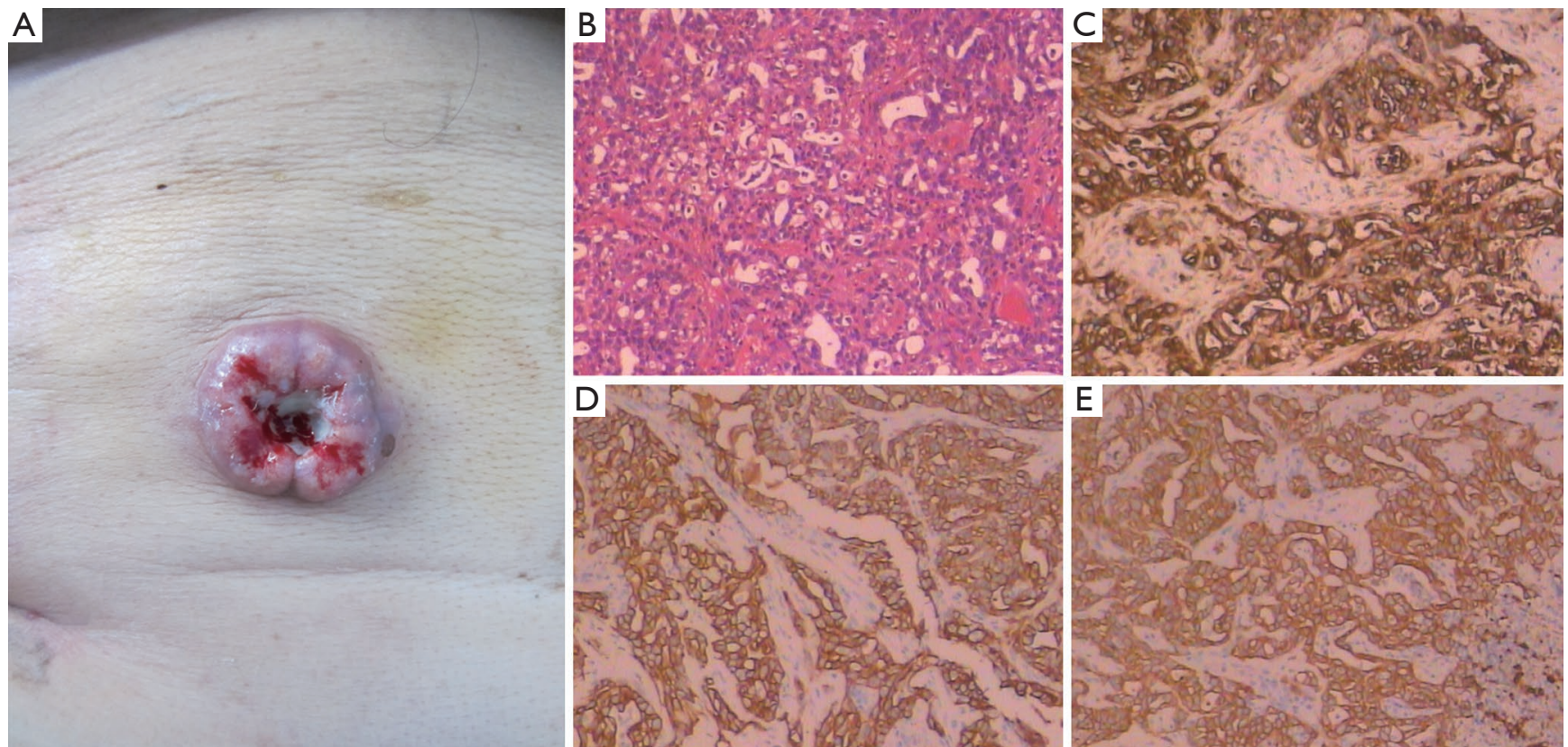

Figure 1 Nodule biopsy. (A) Cutaneous nodule with central ulceration on left upper abdominal wall; (B) hematoxylin-eosin stained biopsy specimen of abdominal wall lesion. Immunohistochemical stains for CEA (C); CK7 (D); and CK19 (E) are positive ( $\times 100)$. CEA, carcinoembryonic antigen; CK, cytokeratin.
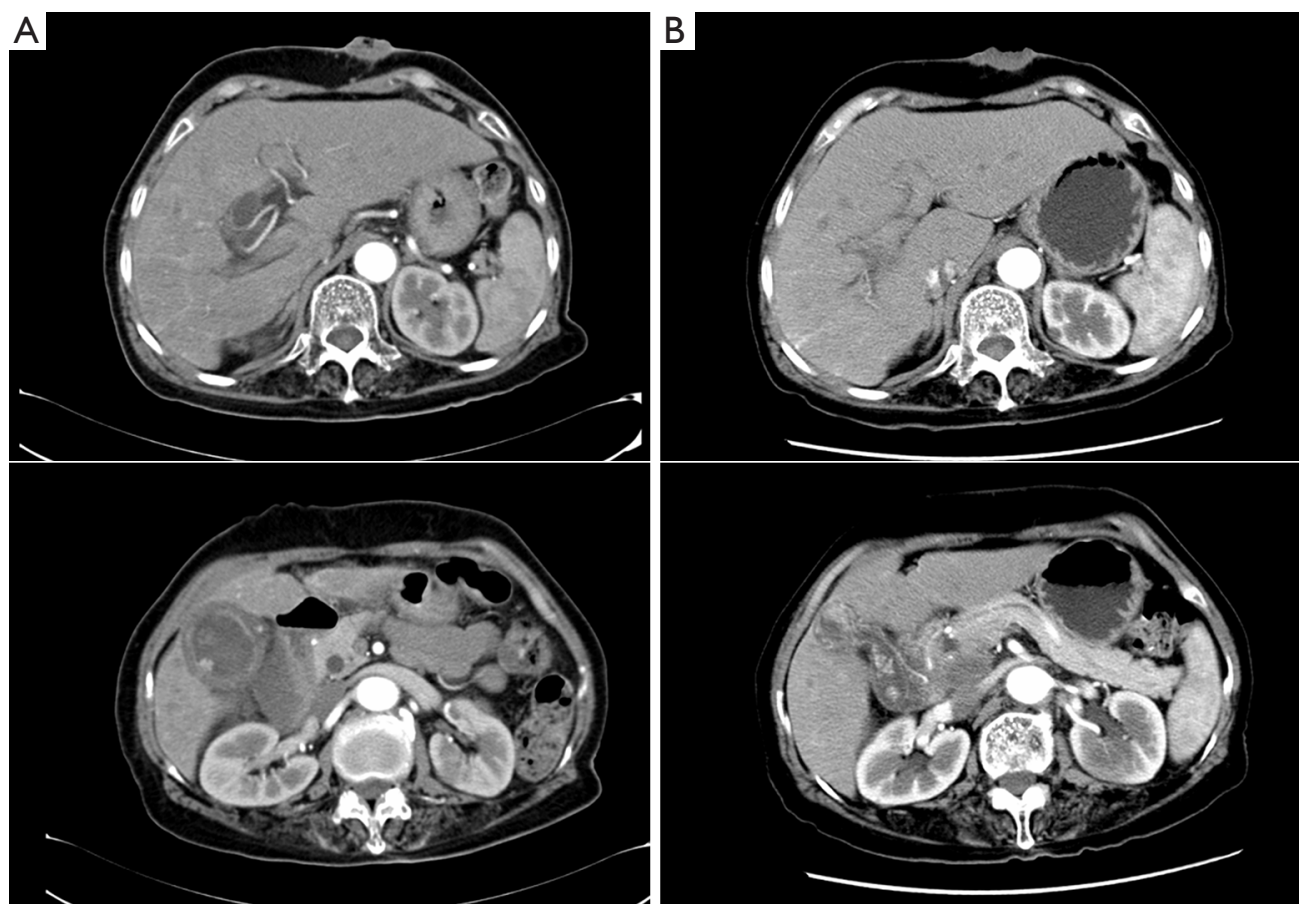

Figure 2 Gallbladder carcinoma: metastatic cutaneous nodule and enlarged lymph nodes in front of the portal vein. (A) Contrast-enhanced CT of abdominal section showed a large occupying lesion in the gallbladder and a metastatic cutaneous nodule; (B) CT showed lesion progression 2 months later. CT, computed tomography. 

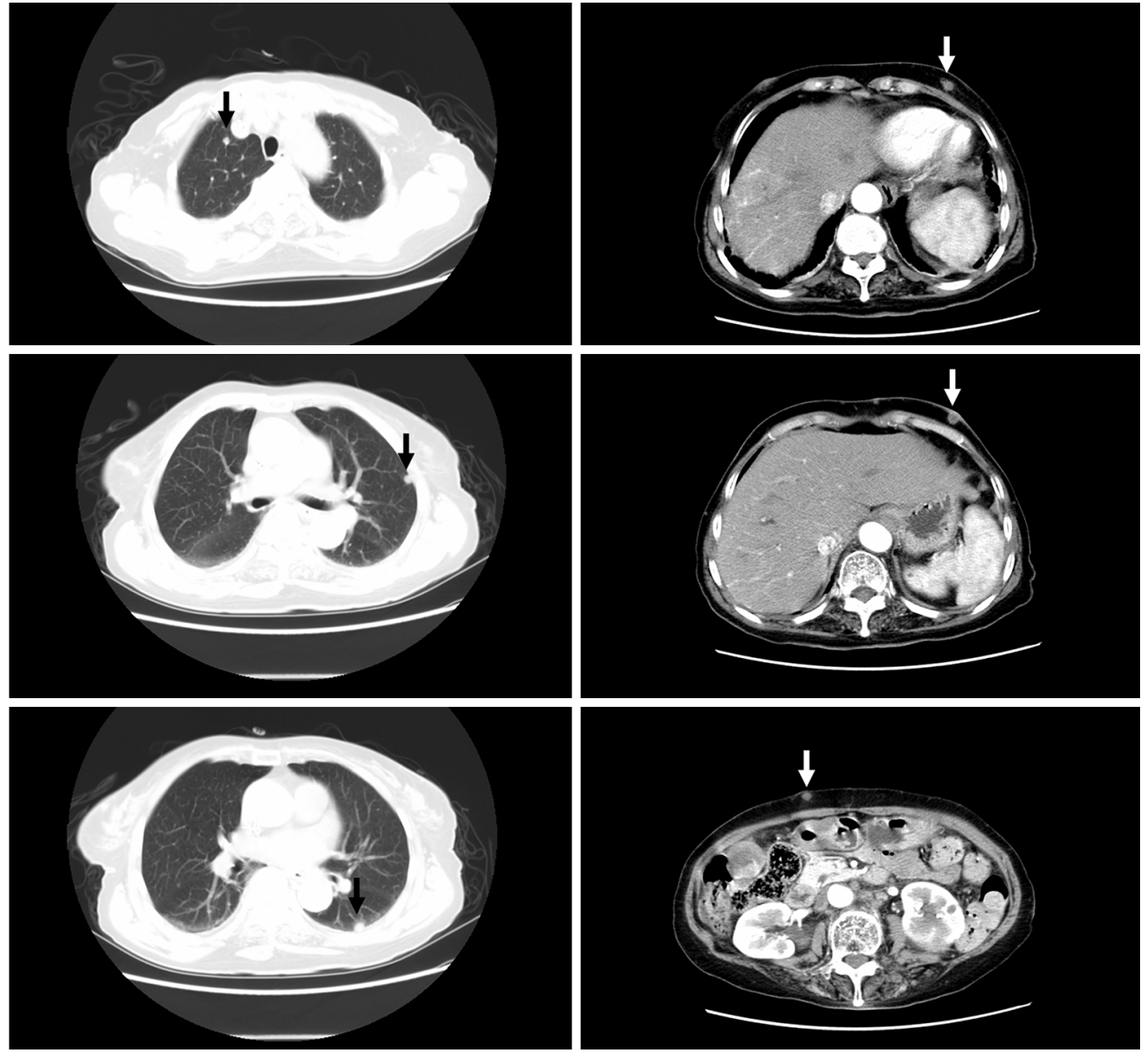

Figure 3 CT scan showed tumor progression with multiple metastatic pulmonary and subcutaneous nodules 4 months later. CT, computed tomography.

metastases from gallbladder cancer stage IV. The patient was treated with two-cycle chemotherapy of gemcitabine, cisplatin, and fluorouracil. Repeat serum alpha fetoprotein (AFP) and CEA progressively increased 2 months later, and CT scan demonstrated disease progression (Figure 2B). Chemotherapy was stopped, and the treatment was switched to tegafur, gimeracil, and oteracil potassium capsules, but CT scan showed tumor progression with multiple metastatic nodule in the lungs and subcutaneous nodules 4 months later (Figure 3). The patient eventually died half a year from diagnosis.

\section{Discussion}

Gallbladder carcinoma, though generally considered rare, is the most common malignancy of the biliary tract, accounting for $80-95 \%$ of biliary tract cancers. An early diagnosis is essential as this malignancy progresses silently with a late diagnosis, often proving fatal. The only likely curative treatments intended for gallbladder carcinoma is usually surgical resection. Unfortunately, only $10-30 \%$ of patients can be considered for surgery, because of loco regional spread or distant metastasis. The comprehensive treatment, such as chemotherapy, radiation treatment and molecular targeted therapy, play important roles in the treatment of gallbladder carcinoma. As for gallbladder carcinoma, loco regional spread is more common than distant metastasis. Metastases usually occur in liver, lymph nodes, adjacent organs and peritoneum. Several mechanisms involving various pathways are implicated in the development of metastases. There are limited examples of cutaneous metastatic gallbladder carcinoma, and the related mechanisms remain unclear.

The overall incidence of cutaneous metastases from 
internal malignancies is roughly $5.3 \%(8)$, and it occurs in nearly all internal malignancies (9). This dissemination can occur through hematogenous, lymphatic, direct or iatrogenic spread. Predominantly skin metastases presents as nodular lesion damaging the dermis and subcutaneous fat, no bigger than $3 \mathrm{~cm}$ diameter and most often asymptomatic. Nevertheless, in more than $46 \%$ of cases, they have atypical presentation. Most times they are very similar to benign lesions. This case highlights the variation that can be exhibited by cutaneous metastatic disease from gallbladder carcinoma. Non-pruritic, indurated lesions in patients should always raise suspicion for cutaneous metastases from internal malignancies and undergo biopsy. Rarely, these lesions may be the initial presentation of malignancy. Cutaneous metastases from gallbladder cancer represent advanced disease and are associated with a very poor prognosis.

\section{Acknowledgments}

Funding: None.

\section{Footnote}

Conflicts of Interest: All authors have completed the ICMJE uniform disclosure form (available at http://dx.doi. org/10.21037/tcr.2017.08.11). The authors have no conflicts of interest to declare.

Ethical Statement: The authors are accountable for all aspects of the work in ensuring that questions related to the accuracy or integrity of any part of the work are appropriately investigated and resolved. All procedures performed in study involving human participants were in accordance with the ethical standards of the institutional and/or national research committee(s) and with the Declaration of Helsinki (as revised in 2013). Written informed consent was obtained from the patient for publication of this manuscript and any accompanying images.

Open Access Statement: This is an Open Access article distributed in accordance with the Creative Commons Attribution-NonCommercial-NoDerivs 4.0 International License (CC BY-NC-ND 4.0), which permits the noncommercial replication and distribution of the article with the strict proviso that no changes or edits are made and the original work is properly cited (including links to both the formal publication through the relevant DOI and the license). See: https://creativecommons.org/licenses/by-nc-nd/4.0/.

\section{References}

1. Hundal R, Shaffer EA. Gallbladder cancer: epidemiology and outcome. Clin Epidemiol 2014;6:99-109.

2. TONGCO RC. Unusual skin metastases from carcinoma of the gallbladder. Am J Surg 1961;102:90-3.

3. Ständer $M$. Skin metastases as a leading symptom in gallbladder neck carcinoma. Z Hautkr 1973;48:537-41.

4. Krunic AL, Chen HM, Lopatka K. Signet-ring cell carcinoma of the gallbladder with skin metastases. Australas J Dermatol 2007;48:187-9.

5. Mukhia R, Shrestha S, Malla K, et al. Cutaneous metastases from carcinoma of gall bladder. JNMA J Nepal Med Assoc 2009 O;48:318-20.

6. Kumar S, Singh D, Goel MM, et al. FNAC site metastasis in gall bladder cancer--a rare presentation. BMJ Case Rep 2012;2012. pii: bcr0220125777.

7. Drouard F, Delamarre J, Capron JP. Cutaneous seeding of gallbladder cancer after laparoscopic cholecystectomy. $\mathrm{N}$ Engl J Med 1991;325:1316.

8. Krathen RA, Orengo IF, Rosen T. Cutaneous metastasis: a meta-analysis of data. South Med J 2003;96:164-7.

9. Bang CH, Bae JM, Kim HS, et al. Recent trends (19912010) of metastatic skin cancers in Korea. J Korean Med Sci 2013;28:1083-8.
Cite this article as: Sun KK, Shen XJ, Zhao H. An unusual case of cutaneous metastases from gallbladder cancer. Transl Cancer Res 2017;6(5):1005-1008. doi: 10.21037/tcr.2017.08.11 